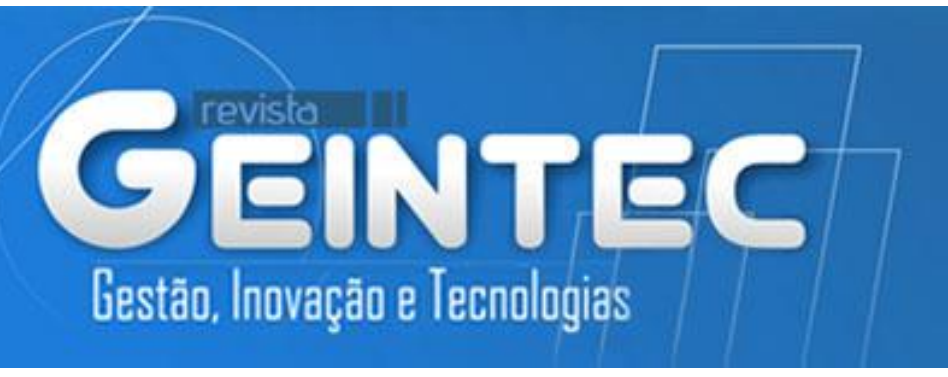

\title{
LÓGICA fuzzy COMO FERRAMENTA PARA AVALIAÇÃo DA DEGRADAÇÃo AMBIENTAL EM RIOS: RELAÇÃO ENTRE OS PARÂMETROS DBO/OD
}

\section{Fuzzy LOGIC AS TOOL FOR EVALUTION OF ENVIRONMENTAL DEGRADATION IN RIVERS: RELATIONSHIP BETWEEN BOD/DO PARAMETERS}

Ada Amélia Sanders Lopes ${ }^{1}$; Silvia Helena Lima dos Santos ${ }^{2}$; Maria Alexsandra de Sousa Rios ${ }^{3}$; Raimundo Oliveira de Souza ${ }^{4}$

${ }^{1}$ Instituto de Engenharias e Desenvolvimento Sustentável- IEDS

Universidade da Integração Internacional da Lusofonia Afro-Brasileira- UNILAB - Redenção/CE - Brasil ada@unilab.edu.br

${ }^{2}$ Instituto de Engenharias e Desenvolvimento Sustentável- IEDS

Universidade da Integração Internacional da Lusofonia Afro-Brasileira- UNILAB - Redenção/CE - Brasil silvia.santos@unilab.edu.br

${ }^{3}$ Departamento de Engenharia Mecânica- DEM

Universidade Federal do Ceará - UFC - Fortaleza/CE - Brasil

alexsandrarios@ufc.br

${ }^{4}$ Departamento de Engenharia Hidráulica e Ambiental - DEHA

Universidade Federal do Ceara - UFC - Fortaleza/CE - Brasil

rsouza@ufc.br

\section{Resumo}

Com base no fenômeno de transferência de massa, a lógica fuzzy desenvolvida no presente estudo estabeleceu relações entre a Demanda Bioquímica de Oxigênio (DBO) e o Oxigênio Dissolvido $(O D)$, para diferentes lançamentos. A aplicação da lógica fuzzy neste cenário, permitiu que as incertezas das variáveis envolvidas no processo, especificamente na solução da equação de advecção, fossem avaliadas e o risco de contaminação de um sistema hídrico diante dos lançamentos de poluentes pudesse ser investigado. No desenvolvimento da lógica fuzzy, os parâmetros de DBO/OD foram convertidos em um conjunto de equações diferenciais $e$ representados como funçõe de pertinência. Para execução das simulações da qualidade da água, os autores utilizaram um programa computacional codificado em linguagem FORTRAN. De acordo com os resultados, concluiu-se que a combinação da Teoria do Transporte de Massa e a da Lógica fuzzy pode ser uma importante ferramenta para a avaliação da Análise de Risco em sistemas hídricos, contribuindo sobremaneira para o monitoramento de temáticas prioritárias da Engenharia Ambiental.

Palavras-chave: Fuzzy; DBO; OD. 


\begin{abstract}
Based on mass transfer phenomena, fuzzy logic developed in this study established relations between the Biochemical Oxygen Demand (BOD) and Dissolved Oxygen (DO), for different releases. The application of fuzzy logic in this scenario allowed the evaluation of uncertainty variables involved in the process, specifically in the solution of advection equation, and the investigation of the contamination risk of a water system before the release of pollutants. In developing of fuzzy logic parameters, $B O D$ / DO were converted into a set of differential equations represented as relevance functions. For water quality simulations, the authors used a computer program coded in FORTRAN. According to the results, it was concluded that the combination of the Theory of Mass Transport and the fuzzy logic could be an important tool for the assessment of risk analysis in water systems, greatly contributing to the monitoring of this priority theme of Environmental Engineering.
\end{abstract}

Keywords: Fuzzy; BOD; DO.

\title{
1. Introdução
}

A avaliação da qualidade da água dos rios, reservatórios ou estuários têm se tornado uma das temáticas de maior interesse para técnicos e pesquisadores das áreas de Recursos Hídricos e Engenharia Ambiental. A crescente demanda por esta temática tem sido causada pelo avanço dos processos degradativos dos corpos hídricos, em consequência principalmente da intervenção do homem e de suas atividades (MCINTYRE e WHEATER, 2004), os quais podem ser observados por meio da divulgação dos indicadores de qualidade das águas.

No que se refere a qualidade dos corpos hídricos, os índices de rios urbanos com baixa presença de vida, de reservatórios com alto risco de eutrofização e de estuários com qualidade ambiental duvidosa, são alguns dos exemplos atuais que se pode encontrar nos mais diversos ambientes aquáticos do mundo. Dentro deste contexto, a aplicação de ferramentas matemáticas computacionais, como por exemplo a lógica fuzzy, direcionadas a tomada de decisões têm contribuído sobremaneira para o avanço das pesquisas em diversos ambientes científicos, obtendose predições valiosas que colaboram para a redução do custo da pesquisa, substituindo muitas vezes a coleta de dados em campo.

Atualmente, com o desenvolvimento da tecnologia digital, modelos matemáticos tornaram-se poderosas ferramentas nos estudos de qualidade ambiental, para meios líquidos e gasosos (LOPES, 2009; CHAPRA, 1997). Nesse sentido, a avaliação da qualidade da água tem sido realizada por duas metodologias que merecem destaque. A primeira diz respeito a um processo de monitoramento direto, para formação de bancos de dados que permitirão a análise criteriosa do estado atual do corpo hídrico (MEYBECK et al., 1996; VANROLLEGHEM et al.,2005) porém, sem boa capacidade de previsão, quando se pretende conhecer tendências com 
respeito ao estado futuro do referido corpo hídrico e a segunda diz respeito à avaliação do risco de falha de um sistema hídrico sujeito a lançamentos de efluentes.

Nesse tipo de estudo, técnicos e pesquisadores lançam mão de métodos probabilísticos para determinar e avaliar as incertezas presentes nas classes de medidas de quantificação, das variáveis determinantes da qualidade ambiental do corpo hídrico (MPIMPAS et al., 2001). Contudo, esta metodologia necessita de robustos bancos de dados para a determinação das funções densidades de probabilidades, os quais quase sempre não são disponíveis, comprometendo a metodologia.

No entanto, com o surgimento da teoria fuzzy nos anos 60, na qual um processo pode ser modelado ainda que haja escassez de dados (DUBOIS e PRADE 1998), a análise de risco tem se tornando uma técnica viável no campo dos recursos hídricos, auxiliando gestores na avaliação quali e quantitativa desse recurso natural. Assim, o presente trabalho apresenta o desenvolvimento de uma metodologia na qual foram combinados os modelos de transporte de poluentes, notadamente o modelo de Demanda Bioquímica de Oxigênio (DBO) e Oxigênio Dissolvido (OD), em rios, com a lógica fuzzy, de modo a calcular o campo de concentração como funções de pertinências e, com isso, determinar o campo de risco causado pela presença de cenários de lançamentos de efluentes. Para determinação do risco e garantia, os parâmetros das equações diferenciais presentes no modelo foram fuzzificados na forma determinística.

\section{Metodologia}

\section{Transporte de Poluentes}

Através da equação de difusão advectiva, o comportamento das concentrações de substâncias poluentes no rio natural pode ser avaliado (JAMES, 1993), Equação 1.

$$
\frac{\partial C}{\partial t}+U \frac{\partial C}{\partial x}=\frac{1}{A} \frac{\partial}{\partial x}\left(E A \frac{\partial C}{\partial x}\right)+S a
$$

Na qual:

$C=$ concentração média em cada seção $\left[M L^{-3}\right]$;

$U=$ velocidade média em cada seção do rio $\left[L T^{-1}\right]$;

$A=$ área da seção transversal $\left[L^{2}\right]$;

$E$ = coeficiente de dispersão longitudinal $\left[L^{2} T^{-1}\right]$;

$S a=$ fonte ou sumidouro de lançamento de substância poluente em um rio $\left[M L^{-3}\right]$.

Desenvolvendo a equação de difusão advectiva, tem-se (Equação 2): 
$\frac{\partial C}{\partial t}+\Psi \frac{\partial C}{\partial x}=E \frac{\partial^{2} C}{\partial x^{2}}-S a$

$\operatorname{Com} \Psi=\left[U-\frac{E}{A} \frac{\partial A}{\partial x}-\frac{\partial E}{\partial x}\right]$

Após o desenvolvimento da Equação 2, aplicou-se as equações da Demanda Bioquímica de Oxigênio (DBO) e de Oxigênio Dissolvido (OD) em um corpo hídrico. Os modelos foram definidos através das Equações 3 e 4:

\section{Equação de DBO:}

$$
\frac{\partial L}{\partial t}+U \frac{\partial L}{\partial x}=E \frac{\partial^{2} L}{\partial x^{2}}-\left(K_{1}+K_{3}\right) L+S a
$$

\section{Equação de OD:}

$$
\frac{\partial L}{\partial t}+U \frac{\partial L}{\partial x}=E \frac{\partial^{2} L}{\partial x^{2}}-K_{1} L+K_{2}\left(C_{S}-C\right)-B
$$

Nas quais:

$L=$ concentração de DBO $\left[M L^{-3}\right]$;

$U=$ velocidade média longitudinal do escoamento $\left[L T^{-1}\right]$;

$S a=$ fonte ou sumidouro $\left[M L^{-3}\right]$;

$K_{1}=$ coeficiente de desoxigenação $\left[T^{-1}\right]$;

$K_{2}=$ coeficiente de reaeração $\left[T^{-1}\right]$;

$K_{3}=$ coeficiente de re-suspensão $\left[T^{-1}\right]$;

$B=$ concentração de oxigênio no processo bentônico $\left[M L^{-3}\right]$;

$E=$ coeficiente de difusão longitudinal $\left[L^{2} T^{-1}\right]$;

$C=$ concentração de oxigênio dissolvido $\left[M L^{-3}\right]$.

\section{Análise de Risco}

Um corpo hídrico que recebe um determinado lançamento de poluentes terá o comportamento desta concentração representado por algumas funções (CHAGAS, 2005), que depende dos parâmetros hidráulico e hidrológico do rio. Entretanto, tais parâmetros não possuem valores precisos em decorrência dos vários processos usados na obtenção destes. Para contornar este problema, surge a lógica fuzzy que permite que tais incertezas possam ser avaliadas nos processos de solução dos modelos matemáticos. 
Segundo Serguieva (2003), a lógica convencional trata as informações de modo binário, classificando-as como verdadeiras ou falsas. Talvez a definição desses dois estados de informação, em alguns casos, seja suficiente, porém, muitas experiências humanas necessitam de uma manipulação mais abrangente do que o simples tratamento de falso ou verdadeiro, sim ou não, certo ou errado. Nesse contexto, a lógica fuzzy (difusa) se torna uma ferramenta apropriada para tratar informações vagas e incertas (LIMA, 2002).

De acordo com Ganoulis et al. (1994), o conceito central da teoria dos números fuzzy baseiase na existência de uma função de pertinência para representar numericamente o grau, por meio do qual, determinado elemento pertence a um conjunto. Assim, conforme Zadeh (1965), um conjunto fuzzy é caracterizado por uma função de pertinência que irá mapear os elementos de um determinado domínio para um número real pertencente ao intervalo $[0,1]$.

Normalmente, uma função de pertinência está na forma $\tilde{A}: \mathrm{X} \rightarrow[0,1]$. Assim sendo, qualquer função representada desta forma pode ser associada a um conjunto fuzzy, dependendo dos conceitos e das propriedades que se deseja representar, considerando-se ainda o contexto no qual o conjunto está inserido. Um conjunto fuzzy é um conjunto de pares ordenados no qual o primeiro elemento é $x \in X$ e o segundo é a função de pertinência $\mu_{\tilde{\mathrm{A}}}(\mathrm{x})$ que mapeia $\mathrm{x}$ no intervalo [0,1]. Assim, a representação de um conjunto fuzzy é matematicamente definida por (Equação 5):

$$
\tilde{A}=\left\{\left(x, \mu_{\tilde{A}}(x)\right) x \in X ; \mu_{\tilde{A}}(x) \in[0,1]\right\}
$$

Na qual:

$\mu_{\tilde{A}}(x)=$ grau de pertinência de $x$ no conjunto $\tilde{A}$

Essas funções representam os níveis de pertinências dos parâmetros físicos, em um processo bem definido. Assim, as incertezas e os riscos ambientais poderão ser analisados e, quanto maior for o grau de pertinência desta variável, maior será o valor da função. O cálculo dessas funções é desenvolvido ao longo do trecho do rio, em cada seção, para diferentes tempos, e assim, deixa de ser um valor único, para se tornar um conjunto de valores fuzzy representando uma função de pertinência.

A concentração e o poder de autodepuração (assimilação) do corpo hídrico foram utilizados como números fuzzy na formulação da metodologia e, foram representados pelas funções de pertinências com valores definidos no intervalo $[0,1]$. O desenvolvimento da modelagem fuzzy necessitou que as equações de DBO e OD fossem reescritas na forma fuzzy, transformando-as em variáveis fuzzy. Para expressar as novas variáveis fuzzy inseriu-se o símbolo “〜” sobre as mesmas. A representação matemática pode ser descrita por (Equações 6 e 7): 


\section{Equação de DBO:}

$$
\frac{\partial \tilde{L}}{\partial t}+\tilde{U} \frac{\partial \tilde{L}}{\partial x}=\tilde{E} \frac{\partial^{2} \tilde{L}}{\partial x^{2}}-\left(\tilde{K}_{1}+\tilde{K}_{3}\right) \tilde{L}+\tilde{S} a
$$

\section{Equação de OD:}

$$
\frac{\partial \tilde{L}}{\partial t}+\tilde{U} \frac{\partial \tilde{L}}{\partial x}=\tilde{E} \frac{\partial^{2} \tilde{L}}{\partial x^{2}}-\tilde{K}_{1} \tilde{L}+\tilde{K}_{2}\left(\tilde{C}_{S}-\tilde{C}\right)-\tilde{B}
$$

Nas quais:

$\tilde{L}=$ representação fuzzy para concentração de DBO $\left[M L^{-3}\right]$;

$\tilde{U}=$ representação fuzzy para velocidade média longitudinal do escoamento $\left[L T^{-1}\right]$;

$\tilde{S} a=$ representação fuzzy para fonte ou sumidouro $\left[M L^{-3}\right]$;

$\tilde{K}_{1}=$ representação fuzzy para coeficiente de desoxigenação $\left[T^{-1}\right]$;

$\tilde{K}_{2}=$ representação fuzzy para coeficiente de reaeração $\left[T^{-1}\right]$;

$\tilde{K}_{3}=$ representação fuzzy para coeficiente de re-suspensão $\left[T^{-1}\right]$;

$\widetilde{B}=$ representação fuzzy para concentração de OD no processo bentônico $\left[M L^{-3}\right]$;

$\widetilde{E}=$ representação fuzzy para coeficiente de difusão longitudinal $\left[L^{2} T^{-1}\right]$;

$\tilde{C}=$ representação fuzzy para concentração de oxigênio dissolvido $\left[M L^{-3}\right]$.

O coeficiente de dispersão pode ser representado por (Equação 8):

$$
\widetilde{E}=0,05937 \frac{\tilde{U} \tilde{A}}{\tilde{S}_{0} B}
$$

Na qual:

$\widetilde{S}_{0}=$ declividade fuzzy do fundo do rio $[L / L]$

$B=$ largura do rio $[L]$

Esse conjunto de equações permite determinar as variáveis dependentes, na forma de funções de pertinência. A metodologia usada para calcular o risco e a garantia fuzzy exige uma comparação entre duas funções de pertinências: 1) Função de Pertinência de Concentração ( $\widetilde{C})$ e 2) Função de Pertinência de Resistência $(\tilde{R})$. A primeira função $(\tilde{C})$ foi utilizada como dados de entrada para o cálculo do campo de concentração ao longo do trecho, para diferentes tempos de exposição a um lançamento. O campo de concentração é calculado pela Equação fuzzy do Transporte de Massa, assim, para cada seção do rio há uma função de pertinência. 
O campo de concentração representa a resposta do sistema hídrico, ou seja, é a capacidade de um determinado corpo hídrico receber cargas poluentes (pontual ou difusa) e de assimilar ou não esta carga. Desta forma, este campo de concentração é de extrema importante no cálculo e avaliação do risco. A segunda função de pertinência $(\widetilde{R})$ representa os níveis máximos de concentrações permitidos para determinados usos e diferentes substâncias presentes nos lançamentos de esgotos, assim, também importante para a avaliação do risco de contaminação de um sistema hídrico.

A diferença entre as duas funções de pertinências, resistência e concentração, define a margem de segurança $(\tilde{M})$ do corpo hídrico (GANOULIS, 1994). O índice fuzzy de falha e de confiabilidade é definido como sendo (Lopes, 2009), Equações 9 e 10:

$$
\begin{gathered}
R_{f}=\frac{\int_{Z<0} \mu_{\tilde{M}}(m) d m}{\int_{Z} \mu_{\tilde{M}}(m) d m} \\
R_{c}=\frac{\int_{Z>0} \mu_{\tilde{M}}(m) d m}{\int_{Z} \mu_{\tilde{M}}(m) d m}
\end{gathered}
$$

Nas quais, $R_{f}$ e $R_{c}$ são funções reais e definidas no intervalo $[0,1]$ e dependem dos parâmetros hidráulicos/hidrológicos, da natureza, da capacidade de escoamento e do tempo.

\section{Resultados e Discussão}

Conforme explicitado anteriormente, o presente estudo trata do desenvolvimento de uma metodologia, por meio da lógica fuzzy, para avaliar a degradação ambiental em rios naturais, utilizando a relação entre os parâmetros de Demanda Bioquímica de Oxigênio (DBO) e de Oxigênio Dissolvido (OD). Para isso, um conjunto de simulações foi realizado tomando-se como base diferentes cenários de lançamentos de poluentes.

Inicialmente foi simulada a condição de um rio natural de sessão retangular, com declividade $0,00005 \mathrm{n} / \mathrm{n}$, rugosidade 0,01 , largura $20 \mathrm{~m}$ e vazão volumétrica de $20 \mathrm{~m}^{3} / \mathrm{s}$. Na simulação foi considerado um canal de $50 \mathrm{~km}$, com lançamentos na origem desse trecho. De acordo com a metodologia do cálculo de risco, necessitou-se do cálculo de uma função marginal de segurança que, representou a diferença entre a função de pertinência da concentração de OD ao longo do rio e a resistência estabelecida em norma para cada tipo de uso. Assim, conhecendo a distribuição do campo de concentração para cada seção do rio e para cada intervalo de tempo, foi possível determinar a função marginal de segurança. 
Na Figura 1 está apresentado o comportamento das funções marginais de segurança, para a simulação tomando-se como base para a resistência, os limites estabelecidos pelas resoluções CONAMA 357/2005 e 412/2009, para rios de classe I e III. Os resultados apresentados são para as seções 6 e 11, a $10 \mathrm{~km}$ do ponto de lançamento.

FIGURA 1 - Funções marginais para as seções 6 e 11, em 8 horas, com C= 300 mg/L

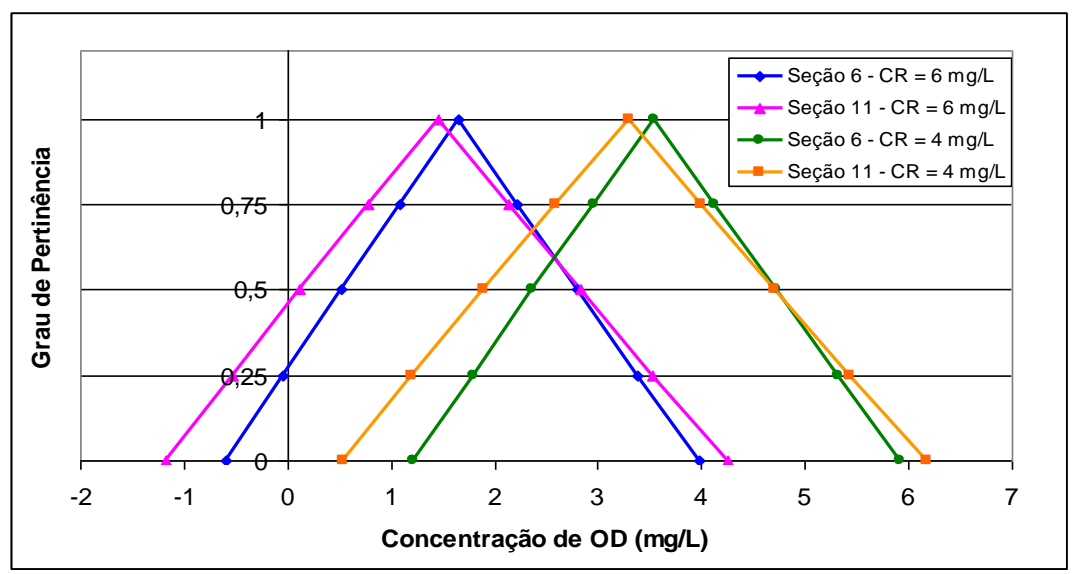

Fonte: Autores

Como podem ser observadas, as simulações tomando-se como base os rios de classe I, tem funções marginais deslocadas para a esquerda, e as seções de classe III têm as suas funções marginais deslocadas para direita. Este resultado mostra que o risco para os rios de classe I são maiores do que para os rios de classe III; e corrobora com as observações, tendo em vista que quanto mais nobre o uso do rio, maior o risco do mesmo em não atender os limites estabelecidos. Este resultado mostra a eficiência da metodologia proposta para o cálculo de risco fuzzy, tomando como base o modelo de DBO/OD "fuzzificado".

Na Figura 2 está apresentada a simulação para uma concentração de 400 mg/L. Os resultados mostram que quando a concentração aumenta, as funções marginais tendem a se deslocar para a esquerda, aumentando o risco de falha.

FIGURA 2 - Funções marginais para as seções 6 e 11, em 8 horas, com C = 400 mg/L 


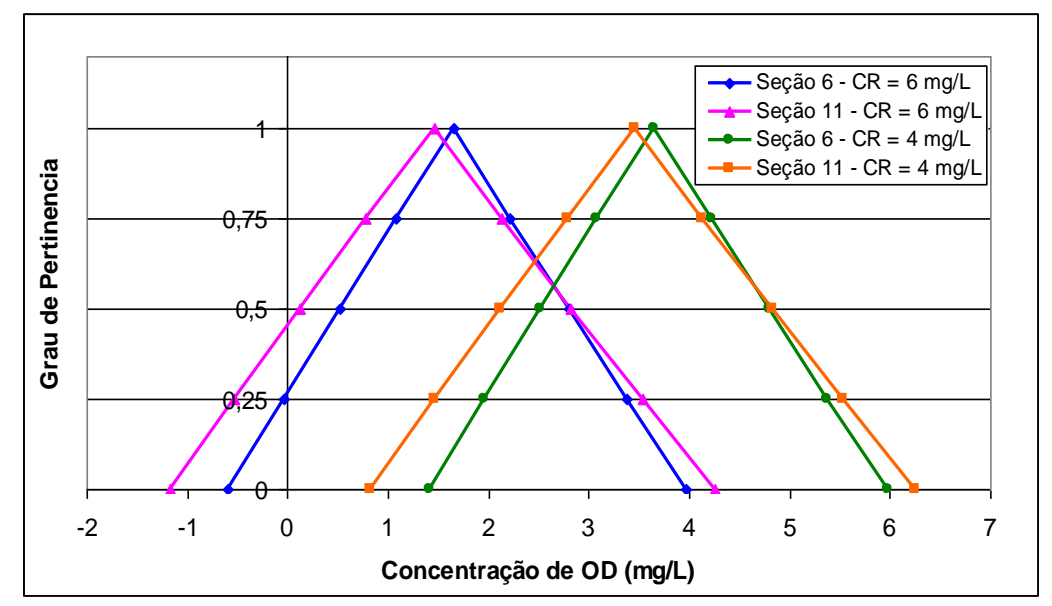

Fonte: Autores

Nesta etapa serão apresentados os resultados das simulações para o cálculo de risco e da garantia, com base na metodologia proposta. No estudo foram verificados diferentes cenários, de modo que se pudesse analisar o comportamento dos campos de risco e da garantia sob a influência desses cenários. Nas Figuras 3 e 4 estão apresentados os resultados da mesma simulação anterior, considerando um rio com uma vazão volumétrica de $10 \mathrm{~m}^{3} / \mathrm{s}$, para um lançamento de $400 \mathrm{mg} / \mathrm{L}$ de DBO, com lançamentos na origem.

De acordo com os resultados, tem-se que, neste caso, onde a vazão do rio é menor, o risco aumenta consideravelmente. Para um tempo de exposição de 10 horas, o risco atinge um valor superior a 50\%, o que é um risco alto para os padrões ambientais de um corpo hídrico. Estes resultados mostram que efluentes domésticos com concentrações de DBO próximas a 300 ou 400 $\mathrm{mg} / \mathrm{L}$, precisam de um tratamento inicial, de modo que sua concentração final cheguem ao rio com valores mais baixos. Do contrário o risco de contaminação do meio aquático será muito grande.

Figura 3 - Risco de degradação em diferentes tempos e vazão volumétrica de $10 \mathrm{~m}^{3} / \mathrm{s}$

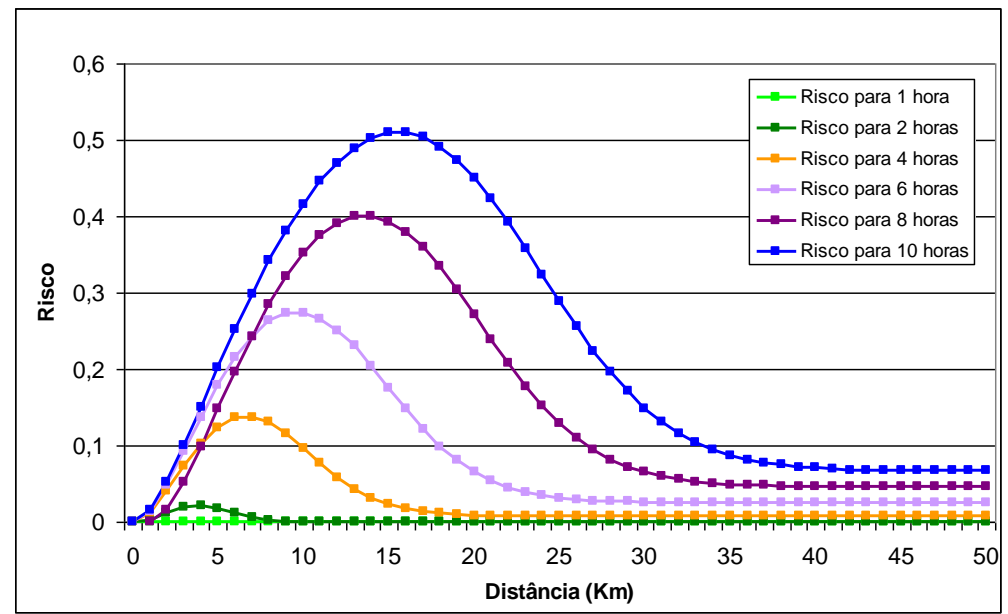

Fonte: Autores 
Figura 4- Garantia contra a degradação para diferentes tempos e vazão volumétrica de $10 \mathrm{~m}^{3} / \mathrm{s}$

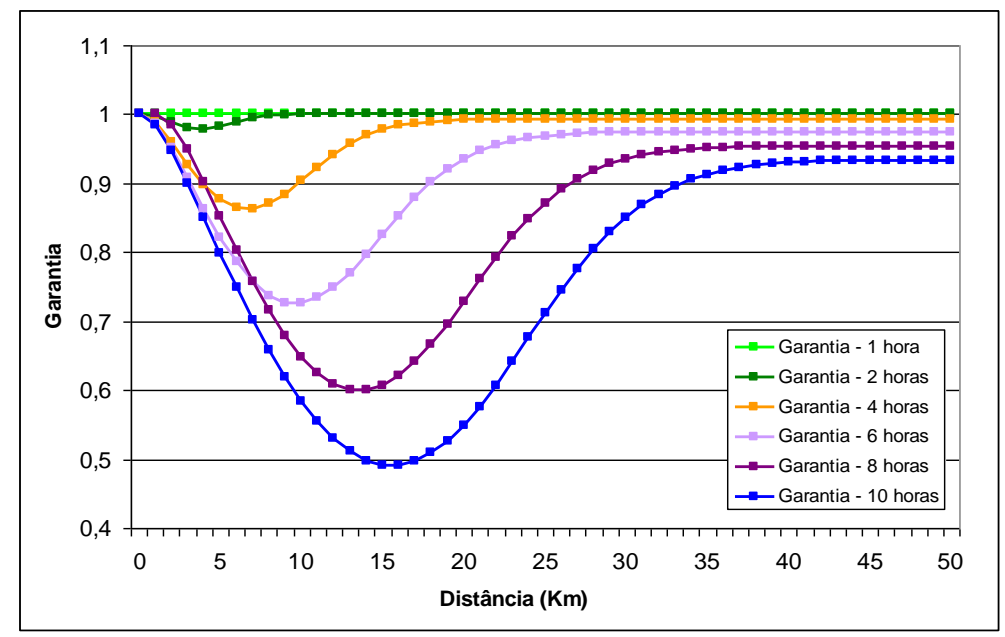

Fonte: Autores

Nesta etapa do desenvolvimento serão apresentados os resultados obtidos a partir de uma simulação que foi realizada tomando-se como base os dados do Rio Cocó, rio urbano que corta a cidade de Fortaleza, no Estado do Ceará. Esta aplicação mostra também a grande vantagem de se usar a Lógica fuzzy para calcular o Risco de Degradação Ambiental em um sistema hídrico, como é o caso de um rio urbano. Neste caso, poucos foram os dados necessários, o que garante a facilidade do uso desta teoria nos estudos de Análise de Risco.

Para esta simulação foram considerados o coeficiente de rugosidade de 0,04 , para o número com maior grau de pertinência, a vazão volumétrica média de $3 \mathrm{~m}^{3} / \mathrm{s}$, a declividade média como sendo 0,00005, a largura média como sendo de $10 \mathrm{~m}$ e possíveis cargas de lançamentos de DBO, que variaram de 200 até $600 \mathrm{mg} / \mathrm{L}$. Todos esses valores foram extraídos de pesquisas prévias, referenciadas anteriormente. Esses valores foram fuzzificados utilizando-se o desvio médio de $20 \%$.

Nas Figuras 5 e 6 estam apresentados os resultados dos campos de risco para os diversos lançamentos, como função da distância. O tempo de observação foi de 12 horas. Como pode ser observado, o rio Cocó, como era de se esperar, devido a baixa vazão volumétrica, não tem capacidade de receber cargas poluentes acima de $200 \mathrm{mg} / \mathrm{L}$, mesmo que a resistência considerada seja para um rio de classe II, uma vez que, neste caso, o risco crítico já atinge um valor próximo de $30 \%$, o que é considerado um quadro de alto risco. Outra observação importante é que, como verificado anteriormente, o campo de risco segue o mesmo perfil da curva de depleção do oxigênio dissolvido. Com isso, pode-se afirmar que este campo é funcional, cujo comportamento depende de várias funções independentes que compõem sua formulação.

FIGURA 5 - Risco de degradação para diferentes concentrações ao longo do canal do rio Cocó 


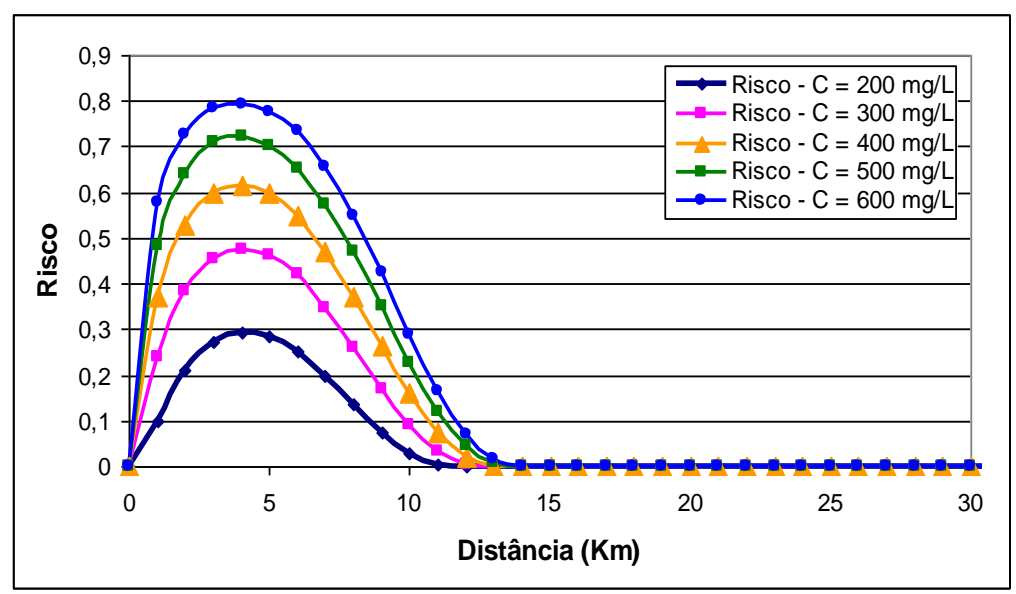

Fonte: Autores

FIGURA 6- Garantia contra a degradação para o rio Cocó, a diferentes concentrações ao longo do canal, a 5 km de origem

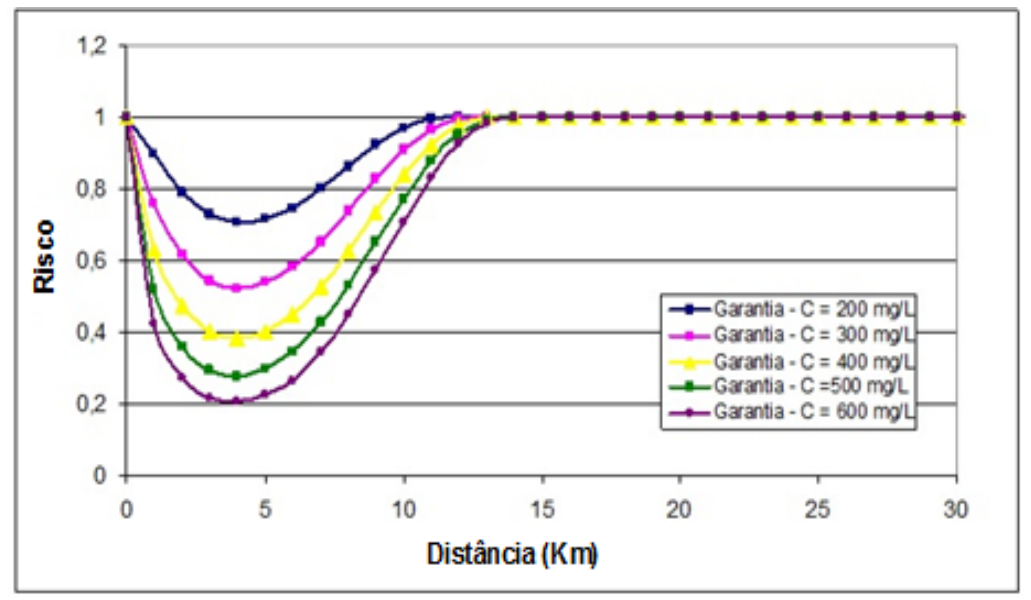

Fonte: Autores

As Figuras 7 e 8 mostram os resultados do risco e da garantia para uma seção tomada a 10 $\mathrm{km}$ do ponto de observação. Os resultados mostram que o risco é zero para as primeiras 3 horas. Somente após este momento, o risco começa a aumentar, atingindo um valor crescente em 12 horas para todos os lançamentos. Desta forma, esse resultado mostra claramente que para um lançamento de $200 \mathrm{mg} / \mathrm{L}$, o risco pode chegar a valores superiores a 50\%, o que representa o indicativo de situação de alto risco, como foi dito anteriormente. Com isso, pode-se concluir que o Rio Cocó, nas condições apresentadas, não pode receber lançamentos de efluentes sem um devido tratamento.

FIGURA 7 - Risco de degradação para diferentes concentrações ao longo do tempo, a10 km da origem 


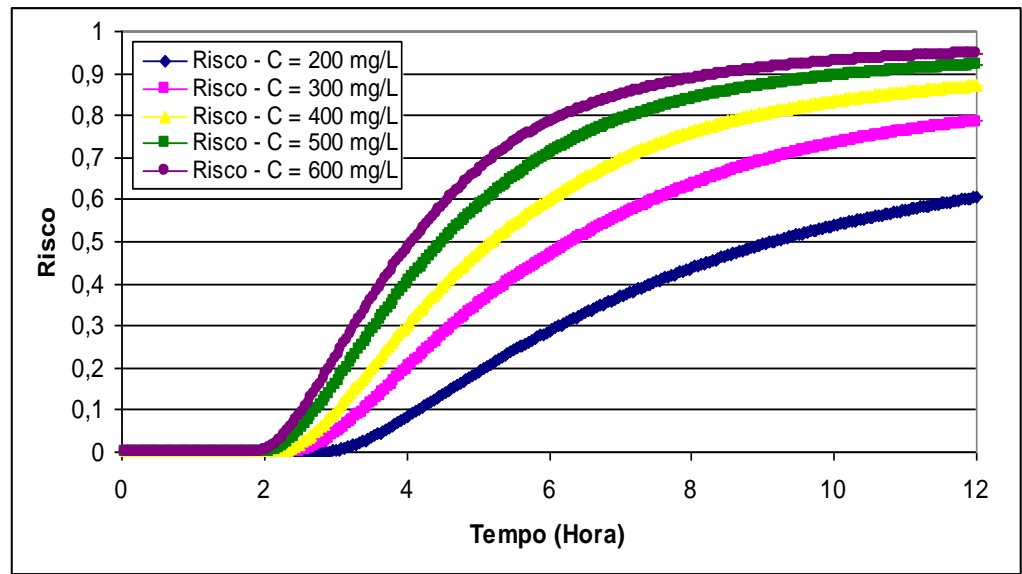

Fonte: Autores

FIGURA 8 - Garantia contra a degradação para diferentes concentrações ao longo do tempo, a 10 km de origem

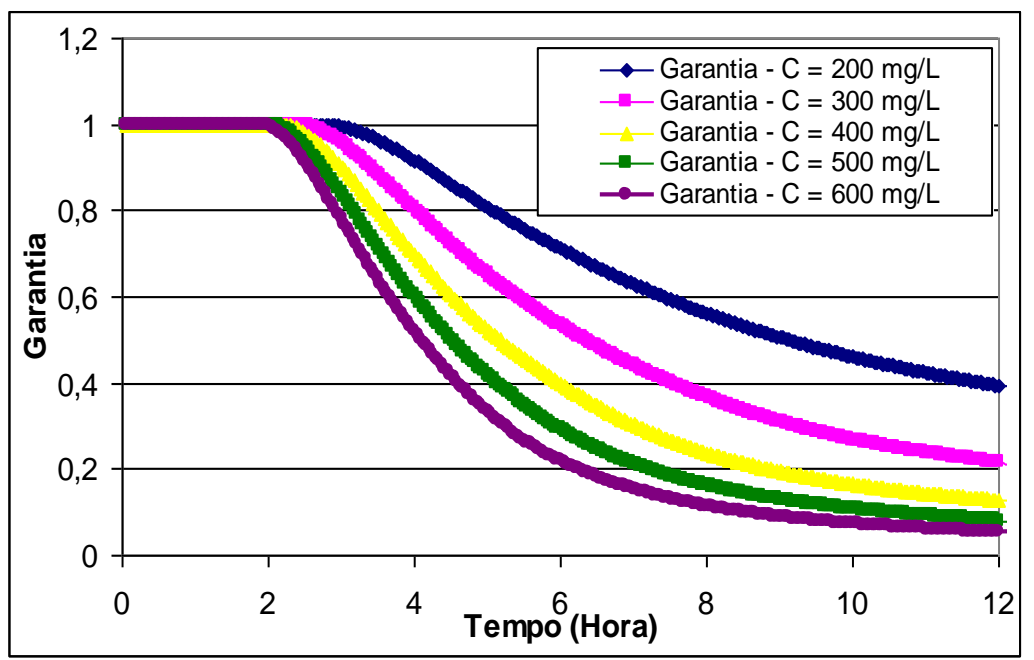

Fonte: Autores

Diante do exposto, pode-se inferir que este estudo serviu para mostrar a importância da Lógica fuzzy, como ferramenta para a Análise de Risco em ambientes aquáticos. É verdade que a fuzzificação das equações diferenciais que determinam os modelos, ainda necessitam de muitos estudos. Ainda há muitos abismos e segredos presentes em cada formulação, tomando como base os diferentes cenários, as condições de contorno, as condições iniciais, enfim, a própria hidrodinâmica presente em cada processo de transporte, que deve ser levada em consideração para o futuro. Mas, não é possível negar que a Teoria fuzzy, com sua formulação especial, com sua simplicidade operacional, pode se tornar uma ferramenta consistente no estudo da Análise de Risco, principalmente, nas questões de Gestão dos Recursos Hídricos pertinentes às concessões de outorgas de lançamentos de efluentes. 


\section{Conclusões}

De acordo com os resultados das simulações, as quais foram executadas para diferentes cenários, levando-se em consideração à hidráulica dos rios, os parâmetros determinantes ao processo de transporte, bem como aos lançamentos de efluentes, com suas características pertinentes às concentrações de DBO é possível concluir que a metodologia proposta com base na Lógica fuzzy, relacionando os parâmetros de DBO/OD apresentou-se como uma ferramenta adequada para o estudo da Análise de Risco em Sistemas Hídricos, sujeitos aos lançamentos de efluentes domésticos ou industriais.

O programa computacional desenvolvido a partir da formulação do Modelo Matemático, mostrou-se eficaz em sua capacidade de produzir resultados. O mesmo permitiu que diferentes fontes de lançamentos pudessem ser avaliadas, bem como, diferentes formas de resultados, tanto do ponto de vista dos modelos determinísticos, como do ponto de vista da modelagem fuzzy, com igual sucesso.

No que se refere aos dados obtidos para o Rio Cocó, os resultados foram bastante significativos e permitiram concluir que rios com este volume hídrico, comuns na região do nordeste brasileiro, não têm capacidade receptora, tomando como base o nível de concentração de DBO presente nos esgotos domésticos. Na simulação executada para várias concentrações de DBO, o risco encontrado foi muito alto e a garantia contra a degradação muito baixa, o que permite concluir que há necessidade de se fazer um tratamento primário nesses efluentes, antes de se tentar qualquer tipo de lançamento. Do contrário, certamente o rio estará fora dos padrões estabelecidos pelas normas que tratam da qualidade ambiental de corpos hídricos.

Finalmente, os resultados permitiram concluir que a combinação entre a Teoria de Transporte de Massa e a Lógica fuzzy pode ser uma importante ferramenta a ser considerada para o estudo de Análise de Risco de sistemas hídricos, contribuindo sobremaneira para o monitoramento de temáticas prioritárias da Engenharia Ambiental.

\section{Referências}

CHAGAS, P.F. Perspectivas da aplicação da teoria fuzzy para cálculo de risco em sistemas hidrodinâmicos. 2005. 204f. Tese (Doutorado em Engenharia Civil) - Programa de Pós-Graduação em Engenharia Civil, Universidade Federal do Ceará, Fortaleza.

CHAPRA, S.C.; In: Surface Water Quality Modeling. McGraw-Hill, New York, 1997.

DUBOIS, D.,PRADE, H., An introduction to fuzzy systems. Clinica Chimica Acta. 270, 3-29, 1998.

GANOULIS, J.G. Engineering risk analysis of water pollution. Probabilities and fuzzy sets. VCH publishers Inc. Weinheim; New York; Basel; Tokyo, 1994. 
JAMES, A. Introduction to water quality modeling, 2.ed. By John Wiley \& Sons, 1993.

LIMA, C.A.A. O uso do modelo QUAL2E na simulação das qualidades das águas do Rio Jacaré-Guaçu. 1997. 168f. Dissertação (Mestrado em Escola de Engenharia de São Carlos). Universidade de São Paulo. São Carlos, São Paulo.

LOPES, A.A.S. Desenvolvimento de uma metodologia com base na teoria fuzzy aplicada a modelos de oxigênio dissolvido (OD) para calcular o risco de degradação ambiental em rios naturais. 2009. 187f. Tese (Doutorado em Engenharia Civil) - Programa de Pós-Graduação em Engenharia Civil, Universidade Federal do Ceará, Fortaleza.

MEINTYRE, N.R.; WHEATER, H.S. A tool for risk based management of surface water quality. Environmental Modeling \& Software. (19), 1131-1140, 2004.

MEYBECK, M. River water quality global ranges, time and space variabilities, proposal for some redefinitions. Verh. Internat. Verein. 26, 1996.

MPIMPAS, H., ANAGNOSTOPOULOS, P., GANOULIS, J., Modeling of water pollution the Thermaikos Gulf with fuzzy parameters. Ecological Modelling. 142, 91-104, 2001.

SERGUIEVA, A.; HUNTER, J.; Fuzzy Interval Methods in Investment Risk Appraisal. Fuzzy Sets and Systems, 142, 443-466, 2003.

VANROLLEGHEM, P.A.; BENEDETTI, L.; MEIRLAEN, J. Modelling and real-time control of the integrated urban wastewater system. Environmental Modeling \& Software. 20, 427-442, 2005.

Recebido: $26 / 06 / 2015$

Aprovado: 01/05/2016 